\title{
Sciendo
}

F

studia humana

QUARTERLY JOURNAL
Studia Humana

Volume 9:3/4 (2020), pp. 194-202

DOI: $10.2478 /$ sh-2020-0037

\section{The Normative Permission and Legal Utterances}

\author{
Marek Zirk-Sadowski \\ University of Łódź \\ Kopcińskiego 8/12 Street \\ 90-232 Lódź, Poland \\ e-mail: msadowski@wpia.uni.lodz.pl
}

\begin{abstract}
:
The author proves that rejecting the existence of permissive norms and limitation of norms to prohibitions and commands alone is possible only with reducing the idea of a function. The essence of the function is then the ability of the expression to generate independently the universal norm formation. Such manipulation is easy on the level of logical analysis, but proves risky from other points of view. If we want the deontic logic, which we construct, to consider the fact that permission is pragmatically necessary for the law-maker to convey his normative preferences, we must solve the consequences of the adopted structure of the function of norms, which originate on the socio-linguistic level. It appears, however, that due to a lack of a pragmatic theory useful for lawyers, there is no proof that the pragmatically strong permission can be expressed by means of a lot of prohibitions and commands (dos and don'ts). Besides, reducing permissions only to the language of legal rules is an obligation to accept the structure of an act of communication, which can find its full motivation in the Husserl's structure of the direct cognition. Keywords: strong permission, permissive norms, behavior control, speech acts, logical analyzes, deontic logics, phenomenology, intentionality, Jan Woleński.
\end{abstract}

\section{Introduction}

In the current theory of law practically only the contention regarding a logical status of rights has managed to reveal the whole complexity of this notion. We omit here the question of the so-called logic of norms, believing that for reason of the assumption of anticognitivism, more convenient could be the consideration of legal inferences within the language of deontic logic. It shall be then remembered, as in essence a discourse on the topic of the logical status of rights related to deontic propositions stating that something is permitted. What is conspicuous here are some gaps within the pragmatics of such logic systems. For this reason, their authors restrict the area of their studies only to the statement that 
using deontic propositions is always relativized to some normative system, but not the whole deontic logic within which we use them [6, p. 117].

With rare exceptions those logic systems use three types of operators: injunction, prohibition and permission. These three operators are a reflection of influences exerted on deontic logic systems modal logic. Usually a necessity was associated with an injunction, impossibility with prohibition and a possibility (or permission) with a right. There are however known systems based on analyses carried out by W. N. Hochfeld which within the deontic logic use a much greater number of operators in the process of translating the content of norms into deontic propositions.

The first remark then, suggested by logical analyses of a right, indicates a fact that the problem of rights appears markedly only in deontic logic systems, which use three operators. We could include within these logic systems also those introducing a fourth operator of an indifferent operation, when the indifferent preceded by the external negation gives a formula: IPdi OP or Fp.

Secondly, an entitlement within these logic systems is treated as the so-called weak permission or the so-called strong permission. The contention on the topic of the status of the logical permission relates mainly to the strong permissions. Regarding the weak permission we could say that it is framed as a non-prohibition. The permission framed as non-injunction would have to include what is prohibited. It appears as an intuitive statement that permission equals a non-prohibition, because it includes the mandatory dimension or the indifferent [6, p. 116]. Within this framework a statement of a type: "I could do what they recommend me to do (what I am commanded to do)" is treated as intuitive, which perhaps should be understood as "I have an entitlement to do, what they recommend me to do (what I am commanded to do").

A question arises as to whether such a framed entitlement does not rather relate to intuitions associated with the notion of competence. Also, a statement that an entitlement includes the dimension of the indifferent is not free from doubts. I have to take as intuitive (obvious) an expression: "I could do what is legally neutral". A permission for such a formula requires however an acceptance of a certain concept of the qualification-driven completeness of the legal system. The permission as a nonprohibition brings then to existence also some interpretative problems. It appears that it has to assume the above demonstrated assumptions.

Logicians accept that a weak permission, or "p is not prohibited", could be stated without referring to the function of behavior control. What suffices is the analysis of the dimension of what is ordered and forbidden. In other words, to state that I am entitled to a certain behavior, in the sense of a weak entitlement what is sufficient for me is a description of the dimension regulated with a prohibition and an injunction.

While the dimension of the weak permission could be relativized till the moment of obtaining the division into the dimension of the injunction and the prohibition, it is not so obvious in the case of the so-called strong permission. Arguments appearing in the literature on behalf of the separation of the strong permission are as follows:

1) the existence of the autonomous elements of a normative system is recognized, regarding different injunctions and prohibitions; an often currently provided example consists of the so-called secondary rules within the construction of law presented by H. L. A. Hart;

2) lawyers experience an intuition that the concepts of "being entitled", or "has a right to" express a particular normative content, which is richer than the content of the non-prohibition.

The arguments provided made the logicians seek a deontic functor which would allow for respecting these two arguments. Let us notice that they do not have the same persuasive power. The first one refers to some assumed construction of the legal system. The deontic logic would then have to be based on the relativization to some theoretical notion of the legal system. The second argument is at least of a linguistic nature, if not even of the philosophical nature. This is because it states that the 
linguistic function of norms includes something more than a prohibition and an injunction and that it includes a strong permission, expressed in the language with the utterance "he/she/it has a right". The first argument follows then a reconstructive attitude towards the legal language, and the second one the descriptivist attitude.

If a logician would like to account for those wants and build a deontic logic including strong permissions, he or she has to accept two assumptions, as was shown by G. H. von Wrighte. First, it is necessary to tell the difference between a normative dimension and the out-of-norms (indifferent) dimension. Second, one would need to assume a possibility of formulating deontic propositions relativized to specific norms of the strong permissive nature.

Based on these assumptions, K. Opałek and J. Woleński attempted to demonstrate that elimination of strong permissions is however necessary [6, p. 121].

Consistently with the above mentioned assumptions they distinguish four dimensions of applying norms, normalizing (or regulating): $\mathrm{O}$ (an injunction), $\mathrm{Ps}$ (a strong permission), $\mathrm{F}$ (prohibition), I (the indifference), of which O, Ps, F belong to the normative dimension, and I to the out-of-norms dimension.

Because within the deontic logic we usually accept a proposition that Op leads to Psp, or the dimension of Ps includes an injunction and "something more", it is an interesting question to pose, as to what dimension $\sim p$ belongs, if $p$ is strongly permitted. The authors answer this question in the following way:

If $\sim \mathrm{p}$ belonged to $\mathrm{O}$, then $\mathrm{p}$ would have to belong to $\mathrm{F}$;

If $\sim p$ belonged to $F$, then $p$ would have to belong to $O$ and then consistently with the thesis that $O p$ leads to Psp, $\mathrm{p}$ would be Ps. It would introduce an interpretative paradox, that one needs to know Op, to state that Psp, If $\sim \mathrm{p}$ would belong to I, then $\mathrm{p}$ would also have to belong to I.

If so, then only accepting that $\sim p$ also belong to Ps could guarantee a separation of Ps. Accepting such a thesis and a statement that Op is a necessary condition of Ps. In relation to the above the authors propose a distinction: between "Ps sensu stricto", which includes the marked dimension (?) and "Ps sensu largo", which includes (O) and (?).

Based on this distinction, they propose a thesis that Ps sensu stricto is an analogon of the indifference (strong indifference), while Ps sensu largo is an analogon of the weak permission. It then leads to the rejection of the thesis that rights are norms. It is a consequence of the accepted by the cited authors conception of the function of behavior control. It follows from their paper that they understand in this way a capacity for expressing autonomous generating of the division of the universe of normalizing [6, p. 124].

The analysis of the logical status of rights or entitlements leads us then to a thesis that within the deontic logic systems using three operators, the question of the entitlements could be reduced to the way of comprehending the function of the act of speech, and particularly the function of behavior control. The logical level of the here presented analysis turns out then to be coming from the relations to linguistic findings, and more precisely speaking - the sociolinguistic level findings. Two competing theses emerge on that level. The first one states: the concept of the function of behavior control does not allow for including the entitlements or rights within the category of norms. Taking such a thesis as valid excludes entitlements or rights from the range of the deontic logic.

The last of the mentioned theses provides us with two possibilities of resolving the question of entitlements or rights: a) delete the entitlements from any interests of lawyers, b) recognize that these are expressions, in which there is a different function (e.g. performative function) built upon the function of behavior control, and only the analysis of the whole multi-layer act of communication could allow for distinguishing speech acts known as entitlements or rights. As it seems, to the authors of an article titled: On the disagreements regarding the so called 'permissive norms' [7, pp. 57-64]. 
Making a choice for one of the above-mentioned theses whose catalogue could be found as insufficient, depends on the accepted socio-linguistic assumptions. The problematic matter is that empirical studies are highly difficult to carry out, and above that, they have to be preceded with some determinations of a philosophical nature.

Within the currently demonstrated discourse what is conspicuous is the acceptance of the opponents of the thesis of an assumption that there exist permissive norms, as to that it is possible to differentiate entitlements only at the level of legal regulations. In other words, an expression "he/she/it has a right" is often an indispensable element of the conventional activity of constituting a legal regulation. This means that a legislator is not able to express with an exact list of injunctions and prohibitions their normative preferences. For instance, in order to protect interests of an owner the legislator would have to formulate a huge number of injunctions and prohibitions, which would include all the possible violations of the law of ownership. Because it is technically impossible, the legislator applies a facilitation providing an owner with a right or an entitlement to use a possession. It is possible only at the level of the legal regulation; if we would like to formulate a norm based on these regulations, then it could be only an injunction or a prohibition, because only they unambiguously determine our way of acting, and so they fulfill the function of behavior control, which is practically a norm. The opponents of the normative characteristics of entitlements or rights simultaneously accept then two theses: 1) an entitlement is logically related to an obligation; 2) permissions are pragmatically indispensable for transferring normative preferences [8, p. 64]. The price for this is the abovementioned linguistic construction, in which a right or an entitlement is not related to a function of behavior control, but a performative function. This follows the fact that the entitlement is only the element of activity of conventionally constituting a legal regulation [7, p. 60].

It appears then that the essence of the matter of contention once again lies within the concept of the linguistic function and specifically it depends on what is understood by the function of behavior control. Accepting a thesis that the essence of the function is the fact of autonomously generating the universe of normalizing, we drastically narrow down the concept of a norm. What strikes us here is also a certain inconsequence. An injunction also does not direct in a certain sense someone's behavior, it only says what must not be done, without positively outlining the behavior.

Determining in such a way the borders of the concept of a norm, we take a reconstructivist stance towards the language. It should be remembered however, that as we have shown, a reconstructionist may also demand to recognize normative characteristics of permissions, for instance referring to the assumed concept of the legal system.

\section{Pragmatic Concept of Legal Norms}

Within the further part of the article we shall try to point out at least a part of the problems, which are brought to life with the pragmatic concept of legal norms. Only solving them will enable us in future to conclude the dilemmas which are created by the so-called permissive norms. As it seems, such questions are revealed by an analysis of the mere pragmatics, and later a concept of the function of behavior control built upon it.

The theory of pragmatics is a part of a general sign theory. It is possible then to talk in relation to it about internal and external effects of accepting a determination of pragmatics. The internal questions relate to associations of the theory of pragmatics with the remaining parts of semiotics, i.e. to syntax and semantics. The external problems relate to associations of pragmatics with other scientific disciplines, which are not included in semiotics [5, pp. 217-245]. Because within the theory of law - as in the case of permitting norms described above - internal problems of pragmatics are usually studied, we shall then try to limit our considerations in the same way. 
Within the logical approach to pragmatic problems we are more interested in the question of the pragmatic language than the question of the mere theory of pragmatics. This significant distinction is useful in understanding the way in which logicians deal with the problem of pragmatics. Within a logic system the notion of pragmatics, or a part of pragmatic language, is related to the requirement of adjusting formal languages to deictic features of natural language. While then within theories of pragmatics - such as the above analyzed theory of Ch. Morris - pragmatics is directly the object of studies, it only has to be reconstructed from pragmatic languages.

The theories built of pragmatics show a tendency to introduce notions taken from behaviorism. Usually they are then some versions of the behavior theory [4, p. 60]. While in studying pragmatic languages built by logicians we state that the here occurring concept of pragmatics is applicable only in analyzing artificial languages, which could be interpreted in small pieces of the natural language. Theories of pragmatics and pragmatic languages use the term "pragmatics" for completely different purposes and only a very general theory of language could combine them. Modern semiotics could not fulfill this task for reason of the lower extent of formalization which it reveals. Therefore, proving a pragmatic equivalence between normalizing including permissions and a given set of injunctions and prohibitions is a doubtful matter. For now such equivalence needs to be a priori assumed.

Lack of an exact determination of pragmatics is the main reason of gaps within the construction of the linguistic function. Such inaccuracies cause a series of astonishing philosophical consequences built from the perspective of pragmatic notion of a legal norm. We shall try to demonstrate them within the next point.

The basic feature of this approach is taking a legal norm as a result of a conventional activity. There is not room to go into detailed analysis of the pragmatic criteria of separation of the expressions of legal language. It is sufficient to state that, at least in the Polish theory of law, they come near to the model of communication suggested by R. Jakobson. It is perhaps also linked with British analytical philosophy, the works of J.L. Austin and Nowell-Smith, i.e. the trend of the so-called multifunctionalists.

Jakobson's model starts from four basis concepts: the act of communication, the context, the function of language, and the concept of the dominating function derived from the latter.

In spite of the evident advantages of this model it causes a number of troubles which are difficult to omit by theoretical manipulations. Treating a legal norm as the effect of a conventional activity is the basic feature of this approach.

We could say that an utterance "a legal norm exists" is equivalent to a statement that by constituting that norm rules of cultural interpretation have been fulfilled, characteristic for the symbolic activity of constituting a legal norm. In other words, the nature of a legal norm is not fully explained with the analysis of the function of behavior control, because its fulfillment decides merely about the normative character of an utterance. A statement that a given norm is a legal norm, requires an analysis of this norm from the perspective of the performative function. A similar statement could be referred to the axiological questions, except for a difference that instead of the function of behavior control we should talk about the expressive function. This type of framing the problem of norms assumes however a normative theory of culture. This means that the culture is created by the total of patterns of behaviors, which was created and perpetuated within the process of social interaction occurring within a given community.

If we realize such nature of the assumed notion of a culture, then an analysis of legal norms reveals certain concerning features. The basic philosophical problem, which was brought to existence by the semantic theory of a norm, was a danger of falling into such philosophical solutions which at the ontological level or epistemological require negating the opposition between the Is-Ought. 
The pragmatic concept of a norm allows for omitting this problem and limiting considerations only to the level of language. Accepting one or a different ontology seems to be neutral for this concept. The semantic theory of a norm requires introducing a concept of a meaning, and so referring to the category of being, if the term of a "meaning" is not used with a persuasive sense only. For it happens that a term of a "meaning" is also used in pragmatic considerations, which has that effect that it is necessary to construct two notions of a meaning. The first one which relates to the semantic relation and the second, which in essence means some feature of a whole act of communication, allowing for identifying an act of communication. A good illustration of this question is the mentioned concept of a permission or a right, in which we could recognize a permission to be a result of an effect of performative function, upon which other elements of an act of communication are built, whose essence is issuing a legal regulation. It is then not about a known distinction between a sense and a meaning, because both these terms refer to a semantic relation, but as if about a certain ideal sense, which unifies an act of communication.

Excluding such persuasive use of a term of a "meaning", applying semantic theory of a norm is related to the risk of disrespecting the difference, which has place at the ontological, epistemological and linguistic level between the Is and the Ought. An advantage of pragmatic construction is to rely on the complete bypassing these issues. Meanwhile, if we look more closely at the construction of rules of cultural interpretation, which decides about our calling some utterance a legal norm, then it is easy to state that the mentioned opposition is not omitted at all, but it is transited to the higher level. Instead of contemplating the problem of obligation at the level of an utterance, it is transferred to analyses related to the concept of a culture. The opposition of a being and - an obligation becomes replaced with the opposition of a culture and nature. Only at this level the mentioned philosophical problem could be resolved. A question then arises as to whether it is then a theoretic-legal problem. It seems that it is rather related to the philosophy of culture. Removing philosophy even from such understood pragmatic theory of a norm is however impossible. Let us try then to consider where the most difficult problem could be found.

We have talked about a double use of the term of a "meaning". If we reject such erroneous theoretic intervention, we will not remove the problem which arises on this occasion. Let us call this question a problem of identifying an act of communication. It appears when we decide to prefer the multifunctionality of an act of communication. Usually it is assumed that an act of communication is able to simultaneously perform several functions. The context in which an act of communication appears decides which of them is the dominant. Depending on the one which dominates within a given context a type of a formulated utterance is determined: a descriptive proposition, a norm, an assessment, etc.

Domination of a function within a given context does not mean however, that the remaining functions disappear. Even if we introduce a distinction of the actual and a potential fulfillment of a function, then still a question arises about how an "identity" of a given act of communication could be found, due to which we would be able to state that we are still considering the same act of communication. It is also from a different point of view a significant question. Within this framework a border between the language and a situation in which it is used is blurring. The language or rather speech appears to be the whole event, while the mere utterance just one of the elements of that event. The question about the potential of identifying an act of communication is then also for this reason significant.

Solving this problem appears to be possible in a few ways. The first depends on recognizing that what sustains an act of communication as a certain entity is a reference function. It somehow constitutes the deepest layer of a studied act, what seems to be a close approximation of that solution are suggestions of Z. Ziembinski [9, p. 115]. In the case of normative utterances difficulties emerge, 
when we ask how the reference function could be fulfilled with the functors of the "I ought to" type. An example is the considered in the previous points problem of an entitlement or a right.

We could obviously assume that they never occur independently, but as parts of an utterance and a notion of fulfilling a function should be referred to the intention of the whole utterance. It is however always necessary to resolve within a proposed solution the problem of criteria of distinguishing a statement of something from describing something. This difficulty we try to omit with a different construction, in which instead of recognizing the reference function as an elementary in some way, an additional notion of a "plot of the utterance" or "a propositional act" is introduced [2, pp. 84-97]. Their theoretic role is interrelated, but because a notion of a propositional act is more precise, we shall try to talk about the currently interesting for us problem by using it.

A propositional act is an act of speech considered only as an indicator of some object and a pronouncement or a judgment regarding relating to some of its features [2, p. 86]. We are not that much interested in the way of pronouncing that feature of an object while studying it. A hypothetical situation of a lack of a propositional act within an act of speech would cause for instance that in expressing a question we would have to separately inform the listener about the content of that question. Appropriately this problem appears within normative utterances. All the functions of an utterance would be therefore superstructured with the so understood propositional act. Consistently with the intention of the cited author it should rather be said that it is about dividing a propositional act and an appropriate illocutionary act. A construction of a propositional act seems to be more persuasive than the earlier proposed solution. Basing the whole act of communication on the reference function (a semantic relation) means in fact a return to the old problem of differentiating a being and an obligation, although, to omit it, a pragmatic analysis was introduced. The notion of a propositional act could not be confronted with this objection, but it raises others, not less complex philosophical problems.

A question arises as to whether an act of speech could be considered a propositional act whether it does not require introducing certain epistemological assumptions. For this act is not an ostensive definition despite that its part is indicating a certain object. A propositional act could refer both to perceptual objects, as well as theoretic, while the ostensive definition is related to perceptual terms. What is then the mentioned 'indication' within determining a propositional act?

Similar questions are posed by 'stating a certain feature'. Consistently with assumptions introduced by the cited author, it is neither a statement nor a description which is a kind of a statement. Where do then features of an object come from and what is the nature of pronouncing them? We could try to assume that it is a certain theoretical construct, the constantly occurring factor or a category of an act of communication. For the author of this construct it is however just an act of speech, and an act of speech is a case of using an utterance. The essence of the problem is included then within the capacity of indicating an object and pronouncing its certain feature in relation to it in a neutral way, i.e. without recognizing the way of pronouncing or judging. The question about the conditions which have to be met to make this stance possible is at the same time a question about philosophical consequences of the construct of a propositional act.

\section{Jacobson's and Husserl's Approaches}

It appears as justified a statement that the full explanation of this type of a construct we could find only within phenomenological contemplations From the theoretic point of view, we could talk about relations of a construct of an act of communication in Jacobson's and Husserl's works. A construct of a pure sense to which a notion of a propositional act is similar is possible to justify only with acceptance of transcendent reductions. A transcendent reduction is a fulfillment of a stance of suspending everything that is external, substantial and revealing or uncovering what is immanent within 
consciousness. Externality is not only empirical reality within the traditional meaning of this word, it is also every real being and so also the psychic being. The starting point within such limited considerations is - according to E. Husserl - consciousness in the sense of Cartesian cogito, understood as any experience of own 'I' within its particular shapes, how: I perceive, I feel, I desire, etc. They are contemplated as a stream of experiences. Every such cogitatio is intentional and it possesses its own essence, which should be captured within its particularity [3, p. 99]. The notion of a propositional act or a plot of an expression could be interpreted as intentionality of an act of speech. More visible becomes this analogy, if we use the developed concept of intentionality, i.e. when we introduce a distinction of two dimensions of an intentional experience: noesis and noema [10, p. 93].

Currently we could say that the construction of a plot of an utterance and of a propositional act could be ordered and assigned to Husserl's notion of noema, the pure sense of an object. If so, then from the pragmatic concept of an utterance, and so also the pragmatic theory of norms, it is possible to derive assumptions whose content appears to be consistent with propositions of E. Husserl's phenomenology. An attempt at escaping philosophical problems, characteristic for the semantic concept of a norm, leads to new philosophical questions which could be linked to concepts assuming the so-called pure consciousness or, in other words, the ideal sense.

Limiting a notion of the function of behavior control to the extent of independently generating the universe of normalizing, though it is easy to carry out at the logical analysis level, it appears as risky from other points of view. If we would like the deontic logic which we construct to account for the fact that a permission is pragmatically indispensable for transferring by the employer their normative preferences, we have to resolve the consequences of the accepted function of behavior control, which arise at the socio-linguistic level. Meanwhile it turns out that for reason of the lack of a useful for a lawyer theory of pragmatics, there is no evidence that pragmatically speaking a strong entitlement or a strong right could be expressed with a list of injunctions and prohibitions. Apart from that limiting permissions only to the language of regulations of the law makes one accept a construction of an act of communication which could find the full justification only within Husserl's construction of the noema.

The normative permission is a good example of difficulties which are brought to existence by pragmatic concept of a norm. It appears that a theory based on the concept of an act of speech fulfilling multiple functions must solve a series of new philosophical questions. The accepted convention of the function of the language has consequences within both a logical analysis of a norm, as well as in studies about conventional activities of law making.

These problems reveal a characteristic way of using logic, which can be called a weak phenomenology. The analytical attitude and the phenomenological attitude are often complementary. However, the phenomenological attitude is not revealed by the lawyers. In particular, the pragmatism of norms hides its phenomenological nature, giving the appearance of a direct knowledge of normativeness.

Contrary to appearances, a weak version of phenomenology is not a direct cognition. It only serves to expose the language of the subject, but the analysis is not intended to get to the reality behind the first language. The purpose of the analysis is to translate these expressions into simpler expressions, but already in metalanguage.

Condemnation to the representationalism makes it impossible to achieve the transparency of the sign and an intentional reference to reality [see 1, ch. 6]. Meanwhile, these are two goals to which Husserl's phenomenology leads. The reversal of the direction of analysis towards the construction of metalanguage as an end in itself causes the original function of cognition, i.e. reaching reality through natural language, to be disrupted. The only goal of the analysis is to transfer the language to higher levels. In this way, the reality built by analysis is created but not recognized. 
The direction should be the opposite, that is, revealing reality, striving for clarity of the first objective language and already directly anchoring it in reality. This is especially important for the practical sciences, which undoubtedly include jurisprudence.

\section{References}

1. Dębowski, J. Bezpośredniość poznania. Spory - dyskusje - wyniki, Uniwersytet Marii CurieSkłodowskiej, Wydział Filozofii i Socjologii, Lublin: 2000.

2. Gizbert-Studnicki, T. Stwierdzenie jako akt mowy, Studia Filozoficzne 3, 1973, pp. 84-97.

3. Husserl, E. Idee I, Warszawa: PWN, 1975.

4. Kutschera von, F. Philosophy of Language, Dordrecht-Holland/Boston-USZA, Reidel Publishing Company, 1975, Synthese Library, vol. 71.

5. Lieb, H. H. Pragmatics and non semiotic disciplines, In Kasher A. (eds) Language in focus, Dordrecht-Boston: 1976, Synthese Library, vol. 89, pp. 217-245.

6.Opałek, K., and J. Woleński. On the so called weak and strong permissions, Philosophical Studies 8, 1974, pp. 115-124.

7. Świrydowicz, K., S. Wronkowska, M. Zieliński, and Z. Ziemski. O nieporozumieniach dotyczących tzw. 'norm zezwalających', Państwo i Prawo 7, 1975, pp. 56-62.

8. Woleński, J. Przyczynek do analizy dozwolenia, Państwo i Prawo 1-2, 1982, pp. 61-64.

9. Ziembiński, Z. Podstawowe problemy prawoznawstwa, PWN, Warszawa 1980.

10. Zirk-Sadowski, M. Powinność i wartość w fenomenologicznej filozofii prawa, Zeszyty Naukowe UŁ 28, 1978, pp. 21-34. 